\title{
The evolution of space curves by curvature and torsion
}

\author{
G Richardson and J R King
}

Division of Theoretical Mechanics, School of Mathematical Sciences, University of Nottingham, Nottingham NG7 2RD, UK

Received 9 May 2002

Published 7 November 2002

Online at stacks.iop.org/JPhysA/35/9857

\begin{abstract}
We apply Lie group based similarity methods to the study of a new, and widely relevant, class of objects, namely motions of a space curve. In particular, we consider the motion of a curve evolving with a curvature $\kappa$ and torsion $\tau$ dependent velocity law. We systematically derive the Lie point symmetries of all such laws of motion and use these to catalogue all their possible similarity reductions. This calculation reveals special classes of law with high degrees of symmetry (and a correspondingly large number of similarity reductions). Of particular note is one class which is invariant under general linear transformations in space. This has potential applications in pattern and signal recognition.
\end{abstract}

PACS numbers: $47.32 . C c, 02.20 .-\mathrm{a}$

Mathematics Subject Classification: 74K05, 22E70

M This article features online multimedia enhancements

\section{Introduction}

The purpose of this work is to investigate the motion of space curves obeying a certain class of isotropic velocity laws. It is well known that curves which have the same curvature and torsion as functions of arclength are similar (i.e. identical up to an arbitrary translation and rotation). It follows that isotropic velocity laws depend upon curvature and torsion, and their derivatives (or integrals) with respect to arclength. Here we restrict ourselves to laws which depend only upon curvature and torsion such that the velocity $v$ of the curve is given by

$$
\boldsymbol{v}=\phi(\kappa, \tau) \kappa \boldsymbol{n}+\beta(\kappa, \tau) \kappa \boldsymbol{b}
$$

where $\phi$ and $\beta$ are arbitrary functions of $\kappa$ and $\tau$, and $\boldsymbol{n}$ and $\boldsymbol{b}$ are, respectively, the principal normal and the binormal to the curve; the extra factors $\kappa$ in this velocity law are retained to make the resulting partial differential equation (PDE) formulation simpler. It is worth remarking that all velocity laws of this form are invariant under rotations and translations in the spatial coordinates and will hence exhibit rich symmetry group structures. The laws given 
by $\phi=\phi(\kappa)$ and $\beta=\beta(\kappa)$ are clearly a subclass of (1) but, because $\kappa$ contains no third derivatives of the position vector with respect to arclength (in contrast to $\tau$ ), they will require special treatment.

In practice, curvature and torsion dependent velocity laws arise in the study of line singularities [1]. These arise in a number of physical systems such as superconducting vortices, scroll waves, disclinations, dislocations and superfluid vortices. Typically, the behaviour of these systems is described by a set of PDEs which exhibit singularities along a set of curves in three-space which are free to move as the system evolves. In many situations, the evolution of the system is governed primarily by the presence of these line singularities and, in such instances, it is useful to derive their law of motion. Typically, this law of motion is coupled to a set of (often linear) field equations. However, there are scenarios where the coupling between the field equations and the velocity law is weak and the motion of the line singularities is predominantly self-induced. Examples of self-induced velocity laws falling into the class (1) include the self-induced motion of a superconducting vortex

$$
v=\kappa n
$$

derived in [5] and the self-induced motion of a superfluid vortex

$$
v=\kappa b
$$

derived in [8]. The self-induced motion of scroll waves in excitable media also falls into this category for certain special classes of these materials, though the general law for the selfinduced motion of scroll waves (derived by Keener [9]) also contains terms with derivatives of the torsion. We note that (3) also applies to small aspect ratio fluid vortices [4, 11]. In addition, we mention the dynamics of twisted rods [10], which have many applications (including the motion of supercoiled DNA) but, like scroll waves, depend upon derivatives of the torsion.

As well as providing an insight into a wide range of physical problems, the investigation of (1) is of interest from a purely mathematical point of view. To illustrate this, we consider the evolution of a planar curve moving according to the law

$$
\boldsymbol{v}=G(\kappa) \boldsymbol{n} .
$$

The special case $G=\kappa^{1 / 3}$ has been studied by Sapiro and Tannenbaum [16] who have demonstrated that the resulting PDE has a rich symmetry group structure (with a correspondingly large class of similarity reductions). Of particular interest is a family of elliptical similarity solutions which, as they shrink, preserve their shape. This shape preserving property has been exploited [16] as the basis of an image processing technique. Subsequent work by Wood [17] classifies the Lie point symmetry groups and the corresponding similarity reductions exhibited by (4) in two dimensions and demonstrates that (i) all such laws are invariant under an arbitrary translation in space and time and a rotation in space, (ii) laws of the form $\boldsymbol{v}=\kappa^{\alpha} \boldsymbol{n}$ (here $\alpha$ is an arbitrary exponent) are, in addition, invariant under a rescaling and (iii) $\boldsymbol{v}=\kappa^{1 / 3} \boldsymbol{n}$ is the only law, of this form, exhibiting more symmetry. The similarity solutions associated with $\boldsymbol{v}=\kappa^{\alpha} \boldsymbol{n}$ are investigated in detail in [17] and numerical solutions are also constructed there. Our aim here is to generalize such classifications to curves evolving in three dimensions according to (1). Since all laws of this form are isotropic, it follows that they are all invariant under arbitrary translations (in space and time) and rotations (in space). In the following, we systematically derive all the continuous symmetries to (1). In particular, we show that laws of the form

$$
\boldsymbol{v}=\kappa^{c+1}(F(\kappa / \tau) \boldsymbol{n}+G(\kappa / \tau) \boldsymbol{b})
$$


(here $c$ is an arbitrary exponent and $F(\cdot)$ and $G(\cdot)$ are arbitrary functions) are, as well as being invariant under translations and rotations, invariant under a rescaling. A special case of this class of law,

$$
\boldsymbol{v}=\left(\frac{\kappa}{|\tau|}\right)^{1 / 3} \boldsymbol{n}
$$

is of particular interest because it exhibits a remarkably rich group structure associated with its invariance under general linear transformations. In this context, we mention that there is some interest in resolving space curves, from fuzzy three-dimensional images, within the image processing community (see, for example, [15]). Such issues arise, for example, in locating the position of a blood vessel (represented by a space curve) from an MRI scan.

In order to reformulate (1) as a PDE, we write the position of the curve as a vector function of time and some parametrization $s$, such that $\boldsymbol{x}=\boldsymbol{q}(s, t)$. In terms of the vector $\boldsymbol{q}$ the velocity law (1) becomes

$$
\boldsymbol{q}_{t}=\phi(\kappa, \tau) \kappa \boldsymbol{n}+\beta(\kappa, \tau) \kappa \boldsymbol{b}-G \boldsymbol{t}
$$

where $t$ is the tangent vector to the curve. Here the function $G$ determines the evolution of the parametrization. If, for example, we wish to identify $s$ with the Cartesian coordinate $z$ and write $\boldsymbol{q}=(u(z, t), v(z, t), z)$ we must ensure that the $z$ component of $\boldsymbol{q}_{t}$ is identically zero. This requires that we choose

$G=\frac{\phi(\kappa, \tau)}{\left(u_{s}^{2}+v_{s}^{2}+w_{s}^{2}\right)^{1 / 2}}\left(\frac{1}{\left(u_{s}^{2}+v_{s}^{2}+w_{s}^{2}\right)^{1 / 2}}\right)_{s}+\frac{\beta(\kappa, \tau)}{\left(u_{s}^{2}+v_{s}^{2}+w_{s}^{2}\right)}\left(u_{s} v_{s s}-v_{s} u_{s s}\right)$

where the subscript denotes the partial derivative. The evolution of the curve is then described by the system

$$
\begin{aligned}
& u_{t}=\frac{\phi(\kappa, \tau) u_{z z}}{\left(1+u_{z}^{2}+v_{z}^{2}\right)}+\frac{\beta(\kappa, \tau)\left(u_{z} v_{z} u_{z z}-\left(1+u_{z}^{2}\right) v_{z z}\right)}{\left(1+u_{z}^{2}+v_{z}^{2}\right)^{3 / 2}} \\
& v_{t}=\frac{\phi(\kappa, \tau) v_{z z}}{\left(1+u_{z}^{2}+v_{z}^{2}\right)}+\frac{\beta(\kappa, \tau)\left(u_{z z}\left(1+v_{z}^{2}\right)-u_{z} v_{z} v_{z z}\right)}{\left(1+u_{z}^{2}+v_{z}^{2}\right)^{3 / 2}}
\end{aligned}
$$

in which

$$
\begin{aligned}
& \kappa=\frac{\left(u_{z z}^{2}+v_{z z}^{2}+\left(u_{z z} v_{z}-u_{z} v_{z z}\right)^{2}\right)^{1 / 2}}{\left(1+u_{z}^{2}+v_{z}^{2}\right)^{3 / 2}} \\
& \tau=\frac{\left(u_{z z} v_{z z z}-u_{z z z} v_{z z}\right)}{\left(u_{z z}^{2}+v_{z z}^{2}+\left(u_{z z} v_{z}-u_{z} v_{z z}\right)^{2}\right)}
\end{aligned}
$$

As we shall demonstrate (and as is to be expected a priori from their geometrical interpretation) all such PDEs are invariant under rotation. It is therefore also useful to formulate the velocity law in terms of polar coordinates. This we do by writing

$$
\boldsymbol{q}=r(\theta, t) \cos \theta \boldsymbol{e}_{x}+r(\theta, t) \sin \theta \boldsymbol{e}_{y}+z(\theta, t) \boldsymbol{e}_{z}
$$

and setting $s=\theta$, which requires

$$
G=\phi(\kappa, \tau) \frac{r\left(r_{\theta} r_{\theta \theta}+z_{\theta} z_{\theta \theta}\right)-r_{\theta}\left(r^{2}+2\left(r_{\theta}^{2}+z_{\theta}^{2}\right)\right)}{r\left(r^{2}+r_{\theta}^{2}+z_{\theta}^{2}\right)^{3 / 2}}+\beta(\kappa, \tau) \frac{r_{\theta} z_{\theta \theta}-z_{\theta} r_{\theta \theta}+r z_{\theta}}{r\left(r^{2}+r_{\theta}^{2}+z_{\theta}^{2}\right)}
$$

so as to ensure that the first two components of (5) give the same relation for $r_{t}$. This results in the following system for $r$ and $z$, 
$r_{t}=\phi(\kappa, \tau) \frac{r r_{\theta \theta}-r^{2}-2 r_{\theta}^{2}}{r\left(r^{2}+r_{\theta}^{2}+z_{\theta}^{2}\right)}+\beta(\kappa, \tau) \frac{r^{2} z_{\theta \theta}-r r_{\theta} z_{\theta}+r_{\theta}\left(r_{\theta} z_{\theta \theta}-z_{\theta} r_{\theta \theta}\right)}{r\left(r^{2}+r_{\theta}^{2}+z_{\theta}^{2}\right)^{3 / 2}}$

$z_{t}=\phi(\kappa, \tau) \frac{r z_{\theta \theta}-2 r_{\theta} z_{\theta}}{r\left(r^{2}+r_{\theta}^{2}+z_{\theta}^{2}\right)}+\beta(\kappa, \tau) \frac{r^{3}-r^{2} r_{\theta \theta}+2 r r_{\theta}^{2}+r z_{\theta}^{2}+z_{\theta}\left(r_{\theta} z_{\theta \theta}-z_{\theta} r_{\theta \theta}\right)}{r\left(r^{2}+r_{\theta}^{2}+z_{\theta}^{2}\right)^{3 / 2}}$

with

$\kappa=\frac{\left[\left(r^{2}-r r_{\theta \theta}+2 r_{\theta}\right)^{2}+\left(r z_{\theta}-r_{\theta \theta} z_{\theta}+r_{\theta} z_{\theta \theta}\right)^{2}+\left(r z_{\theta \theta}-2 r_{\theta} z_{\theta}\right)^{2}\right]^{1 / 2}}{\left(r^{2}+r_{\theta}^{2}+z_{\theta}^{2}\right)^{3 / 2}}$

$\tau=\frac{z_{\theta \theta \theta}\left(r^{2}+2 r_{\theta}^{2}-r r_{\theta \theta}\right)-z_{\theta \theta}\left(r^{2}+2 r_{\theta}^{2}-r r_{\theta \theta}\right)_{\theta}+z_{\theta}\left(3 r_{\theta \theta}^{2}-2 r_{\theta} r_{\theta \theta \theta}+6 r_{\theta}^{2}-4 r r_{\theta \theta}+r^{2}\right)}{\left(r^{2}-r r_{\theta \theta}+2 r_{\theta}\right)^{2}+\left(r z_{\theta}-r_{\theta \theta} z_{\theta}+r_{\theta} z_{\theta \theta}\right)^{2}+\left(r z_{\theta \theta}-2 r_{\theta} z_{\theta}\right)^{2}}$.

This formulation is a more convenient one for studying some of the similarity solutions discussed later.

The methods we use to derive the Lie point symmetries (and the similarity reductions) of PDEs (6)-(9), and hence of the velocity law (1), are described in [2, 3, 6]. A more rigorous treatment of the subject can be found in Olver [12].

In the next two sections, we systematically derive the Lie point symmetries of all velocity laws of the form (1) using their PDE formulation (6)-(7). We show that such laws fall into one of three classes, having varying degrees of symmetry, and derive the global transformations under which each class of velocity law is invariant. We use the Lie point symmetries, in section 4 , to catalogue the similarity reductions for each class of velocity law. Finally, in section 5, we draw our conclusions.

\section{Local Lie groups}

\subsection{Formulation}

We apply the standard Lie group method to determine the point symmetries of equations (6)-(9) (see [6], for example) by seeking infinitesimal transformations of the form

$$
\begin{array}{rlrl}
t^{*} & \sim t+\epsilon T(z, t, u, v) & u^{*} & \sim u+\epsilon U(z, t, u, v) \\
v^{*} & \sim v+\epsilon V(z, t, u, v) & z^{*} \sim z+\epsilon Z(z, t, u, v)
\end{array}
$$

where $\epsilon \ll 1$. In addition, we must in the usual way consider the effect of this transformation on the derivatives of the dependent variables $u$ and $v$ with respect to the independent variables $z$ and $t$. We write

$$
\begin{array}{ll}
u_{z^{*}}^{*} \sim u_{z}+\epsilon U^{z} & u_{z^{*} z^{*}}^{*} \sim u_{z z}+\epsilon U^{z z} \\
u_{z^{*} z^{*} z^{*}}^{*} \sim u_{z z z}+\epsilon U^{z z z} & u_{t^{*}}^{*} \sim u_{t}+\epsilon U^{t} \quad \text { as } \epsilon \rightarrow 0
\end{array}
$$

and similarly for the derivatives of $v$. The prolongations $U^{t}, U^{z}, U^{z z}$ and $U^{z z z}$ can be expressed in the standard way (by using the chain rule) in terms of derivatives of $u$ and $v$ with respect to $z$ and $t$,

$$
\begin{aligned}
U^{t} & =\frac{D U}{D t}-u_{z} \frac{D T}{D z}-u_{t} \frac{D T}{D t} \\
U^{z} & =\frac{D U}{D z}-u_{z} \frac{D Z}{D z}-u_{t} \frac{D T}{D z}
\end{aligned}
$$




$$
\begin{aligned}
U^{z z} & =\frac{D^{2} U}{D z^{2}}-2 u_{z z} \frac{D Z}{D z}-\left(u_{z} \frac{D^{2} Z}{D z^{2}}+2 u_{z t} \frac{D T}{D z}+u_{t} \frac{D^{2} T}{D z^{2}}\right) \\
U^{z z z} & =\frac{D^{3} U}{D z^{3}}-\left(3\left(\frac{D Z}{D z} u_{z z z}+\frac{D T}{D z} u_{z z t}+\frac{D^{2} T}{D z^{2}} u_{z t}+\frac{D^{2} Z}{D z^{2}} u_{z z}\right)+u_{z} \frac{D^{3} Z}{D z^{3}}+u_{t} \frac{D^{3} T}{D z^{3}}\right)
\end{aligned}
$$

where

$$
\begin{aligned}
& \frac{D U}{D t}=U_{t}+U_{u} u_{t}+U_{v} v_{t} \quad \frac{D U}{D z}=U_{z}+U_{u} u_{z}+U_{v} v_{z} \\
& \frac{D^{2} U}{D z^{2}}=U_{z z}+U_{u} u_{z z}+U_{v} v_{z z}+2\left(U_{u z} u_{z}+U_{v z} v_{z}\right)+\left(U_{u u} u_{z}^{2}+2 U_{u v} u_{z} v_{z}+U_{v v} v_{z}^{2}\right) \\
& \frac{D^{3} U}{D z^{3}}=U_{z z z}+ \\
& +\left(U_{u z z} u_{z}+U_{v z z} v_{z}\right)+3\left(U_{u u z} u_{z}^{2}+2 U_{u v z} u_{z} v_{z}+U_{v v z} v_{z}^{2}\right) \\
& +\left(U_{u u u} u_{z}^{3}+3 U_{u u v} u_{z}^{2} v_{z}+3 U_{u v v} u_{z} v_{z}^{2}+U_{v v v} v_{z}^{3}\right)+3\left(U_{u z} u_{z z}+U_{v z} v_{z z}\right) \\
& +3\left(U_{u u} u_{z} u_{z z}+U_{u v}\left(u_{z z} v_{z}+u_{z} v_{z z}\right)+U_{v v} v_{z} v_{z z}\right)+U_{u} u_{z z z}+U_{v} V_{z z z} .
\end{aligned}
$$

The terms $V^{z}, T^{z}, Z^{z}$ etc can be calculated similarly, so for example $V^{z z}$ is found by replacing $U$ by $V$ in (18) and making the transformation $u \rightarrow v, v \rightarrow u$. We also need to consider the effect of the transformation on the curvature and torsion; under (14) these go to

$$
\kappa^{*} \sim \kappa+\epsilon K \quad \tau^{*} \sim \tau+\epsilon \mathfrak{T}
$$

where

$$
\begin{aligned}
& K=-3 \kappa \frac{u_{z} U^{z}+v_{z} V^{z}}{M^{2}} \\
& +\kappa\left[\frac{u_{z z} U^{z z}+v_{z z} V^{z z}+\left(u_{z} v_{z z}-v_{z} u_{z z}\right)\left(U^{z} v_{z z}-v_{z} U^{z z}+u_{z} V^{z z}-V^{z} u_{z z}\right)}{u_{z z}^{2}+v_{z z}^{2}+\left(u_{z} v_{z z}-v_{z z} u_{z z}\right)^{2}}\right] \\
& \mathfrak{T}=\tau\left\{\frac{U^{z z} v_{z z z}+u_{z z} V^{z z z}-V^{z z} u_{z z z}-v_{z z} U^{z z z}}{u_{z z} v_{z z z}-v_{z z} u_{z z z}}\right\} \\
& -2 \tau\left\{\frac{u_{z z} U^{z z}+v_{z z} V^{z z}+\left(v_{z} u_{z z}-u_{z} v_{z z}\right)\left(V^{z} u_{z z}+v_{z} U^{z z}-U^{z} v_{z z}-V^{z z} u_{z}\right)}{u_{z z}^{2}+v_{z z}^{2}+\left(v_{z} u_{z z}-u_{z} v_{z z}\right)^{2}}\right\}
\end{aligned}
$$

Substituting (14) and (15) into (6)-(9) and equating terms of order $\epsilon$ yields

$$
\begin{array}{rl}
-U^{t} M^{3}-3 u_{t} & M\left(u_{z} U^{z}+v_{z} V^{z}\right)+M \phi U^{z z}+\frac{\phi u_{z z}}{M}\left(u_{z} U^{z}+v_{z} V^{z}\right)+M u_{z z}\left(K \frac{\partial \phi}{\partial \kappa}+\mathfrak{T} \frac{\partial \phi}{\partial \tau}\right) \\
+ & \beta\left(v_{z} u_{z z} U^{z}+u_{z} u_{z z} V^{z}+u_{z} v_{z} U^{z z}-2 u_{z} v_{z z} U^{z}-\left(1+u_{z}^{2}\right) V^{z z}\right) \\
+ & \left(K \frac{\partial \beta}{\partial \kappa}+\mathfrak{T} \frac{\partial \beta}{\partial \tau}\right)\left(u_{z} v_{z} u_{z z}-v_{z z}\left(1+v_{z}^{2}\right)\right)=0 \\
-V^{t} M^{3}-3 v_{t} & M\left(u_{z} U^{z}+v_{z} V^{z}\right)+M \phi V^{z z}+\frac{\phi v_{z z}}{M}\left(u_{z} U^{z}+v_{z} V^{z}\right)+M v_{z z}\left(K \frac{\partial \phi}{\partial \kappa}+\mathfrak{T} \frac{\partial \phi}{\partial \tau}\right) \\
+ & \beta\left(\left(1+v_{z}^{2}\right) U^{z z}+2 u_{z z} v_{z} V^{z}-v_{z} v_{z z} U^{z}-u_{z} v_{z z} V^{z}-u_{z} v_{z} V^{z z}\right) \\
+ & \left(K \frac{\partial \beta}{\partial \kappa}+\mathfrak{T} \frac{\partial \beta}{\partial \tau}\right)\left(u_{z z}\left(1+v_{z}^{2}\right)-u_{z} v_{z} v_{z z}\right)=0 .
\end{array}
$$

In order that these equations be satisfied for all solutions to (6)-(9), we require that the coefficients multiplying derivatives of $u$ and $v$ be equal to zero. However, care has to be taken 
in doing this since, as usual, the time derivatives are related to the spatial ones by the governing equations and $\kappa, \tau, U^{z}, V^{z}$ etc all depend upon the various spatial derivatives of $u$ and $v$.

We start by setting the coefficients of $u_{z z t}$ and $v_{z z t}$ equal to zero; this implies $T=T(t)$. We next look at the coefficients of the remaining third-order derivatives (i.e. $u_{z z z}, v_{z z z}$ ). These only appear in (20)-(21) through $\mathfrak{T}$ and $\tau$. Since at this stage we are taking $\beta$ and $\phi$ to be functions of $\tau$, it is convenient to rewrite $v_{z z z}$, where it appears in $\mathfrak{T}$, in terms of $\tau$ and $u_{z z z}$. We then equate terms in $u_{z z z}$ to zero to find

$$
\begin{array}{lll}
U_{v v}=0 & U_{v z}=0 & U_{z z}=0 \\
V_{u u}=0 & V_{u z}=0 & V_{z z}=0 \\
Z_{u u}=0 & Z_{u v}=0 & Z_{v v}=0 \\
U_{u z}=V_{v z} & U_{u v}=Z_{z v} & V_{v u}=Z_{z u} \\
U_{u u}=2 Z_{u z} & V_{v v}=2 U_{u v} & Z_{z z}=2 V_{v z} .
\end{array}
$$

Proceeding with the remainder of the calculation, we find in addition that

$$
\begin{array}{lll}
\beta\left(V_{z}+Z_{v}\right)=0 & \beta\left(V_{u}+U_{v}\right)=0 & \beta\left(U_{z}+Z_{u}\right)=0 \\
\left(\kappa \phi_{\kappa}-2 \tau \phi_{\tau}\right)\left(V_{z}+Z_{v}\right)=0 & \left(\kappa \phi_{\kappa}-2 \tau \phi_{\tau}\right)\left(V_{u}+U_{v}\right)=0 & \left(\kappa \phi_{\kappa}-2 \tau \phi_{\tau}\right)\left(U_{z}+Z_{u}\right)=0 \\
\left(2 \phi+3 \kappa \phi_{\kappa}\right)\left(V_{z}+Z_{v}\right)=0 & \left(2 \phi+3 \kappa \phi_{\kappa}\right)\left(V_{u}+U_{v}\right)=0 & \left(2 \phi+3 \kappa \phi_{\kappa}\right)\left(U_{z}+Z_{u}\right)=0 \\
\left(\kappa \phi_{\kappa}-2 \tau \phi_{\tau}\right)\left(U_{u}-Z_{z}\right)=0 & \left(\kappa \phi_{\kappa}-2 \tau \phi_{\tau}\right)\left(U_{u}-V_{v}\right)=0 & \left(\kappa \phi_{\kappa}-2 \tau \phi_{\tau}\right)\left(V_{v}-Z_{z}\right)=0 \\
\left(2 \phi+3 \kappa \phi_{\kappa}\right)\left(U_{u}-Z_{z}\right)=0 & \left(2 \phi+3 \kappa \phi_{\kappa}\right)\left(U_{u}-V_{v}\right)=0 & \left(2 \phi+3 \kappa \phi_{\kappa}\right)\left(V_{v}-Z_{z}\right)=0 \\
\beta\left(U_{u}-Z_{z}\right)=0 & \beta\left(U_{u}-V_{v}\right)=0 & \beta\left(V_{v}-Z_{z}\right)=0 \\
Z_{t}=0 & U_{t}=0 & V_{t}=0 \\
\phi T_{t}=\frac{1}{3}\left(U_{u}+V_{v}+Z_{z}\right)\left(2 \phi+\kappa \phi_{\kappa}+\tau \phi_{\tau}\right) & \beta T_{t}=\frac{1}{3}\left(U_{u}+V_{v}+Z_{z}\right)\left(2 \beta+\kappa \beta_{\kappa}+\tau \beta_{\tau}\right) .
\end{array}
$$

In the case where both $\partial \phi / \partial \tau$ and $\partial \beta / \partial \tau$ are identically zero, we cannot use the argument given above (concerning the third-order derivatives of $u$ and $v$ ) to infer (22) and must therefore perform a separate calculation to find the infinitesimal symmetries. This is considerably simpler than the general case and results in the following relations,

$$
\begin{array}{lll}
T=T(t) & V_{v}=Z_{z} & U_{u}=Z_{z} \\
V_{z}+Z_{v}=0 & V_{u}+U_{v}=0 & U_{z}+Z_{u}=0 \\
U_{t}=0 & V_{t}=0 & Z_{t}=0 \\
\beta T_{t}=Z_{z}\left(2 \beta+\kappa \beta_{\kappa}\right) & \phi T_{t}=Z_{z}\left(2 \phi+\kappa \phi_{\kappa}\right) &
\end{array}
$$

together with the same conditions on the second derivatives as before, namely (22). This special case will be discussed further in sections 2.2 and 2.3. We now divide the analysis into three sub-cases.

\subsection{Case I: arbitrary $\phi$ and $\beta$}

If we allow $\phi$ and $\beta$ to be any function of $\kappa$ and $\tau$ then, from (23), we require

$$
T_{t}=Z_{z}=U_{u}=V_{v}=0 .
$$

The corresponding symmetry group has seven parameters and takes the form

$$
T=t_{0}
$$




$$
\left(\begin{array}{l}
U \\
V \\
Z
\end{array}\right)=\left(\begin{array}{ccc}
0 & h & -f \\
-h & 0 & -g \\
f & g & 0
\end{array}\right)\left(\begin{array}{l}
u \\
v \\
z
\end{array}\right)+\left(\begin{array}{l}
u_{0} \\
v_{0} \\
z_{0}
\end{array}\right) .
$$

We note that $t_{0}, u_{0}, v_{0}$ and $z_{0}$ represent translational invariants and $h, f$ and $g$ represent rotational invariants, so the symmetry can be predicted in advance from the nature of the velocity law (1) but is far from obvious from the PDE formulation. In the special case where $\phi=\phi(\kappa)$ and $\beta=\beta(\kappa)$ we retrieve the same result from (24).

Looking at the equations contained in (23) we see that if $\phi$ and $\beta$ are chosen appropriately we might expect to obtain equations with a higher degree of symmetry. Thus the condition

$$
T_{t}=\left(U_{u}+V_{v}+Z_{z}\right)=0
$$

holds unless

$$
\frac{1}{\phi}\left(2 \phi+\kappa \phi_{\kappa}+\tau \phi_{\tau}\right)=2+c \quad \frac{1}{\beta}\left(2 \beta+\kappa \beta_{\kappa}+\tau \beta_{\tau}\right)=2+c
$$

where $c$ is an arbitrary constant. Similarly, the conditions

$$
V_{z}=-Z_{v} \quad V_{u}=-U_{v} \quad U_{z}=-Z_{u} \quad U_{u}=V_{v}=Z_{z}
$$

hold unless $\phi$ and $\beta$ satisfy

$$
\kappa \phi_{\kappa}-2 \tau \phi_{\tau}=0 \quad 2 \phi+3 \kappa \phi_{\kappa}=0 .
$$

Similarly in the special case $\phi=\phi(\kappa)$ and $\beta=\beta(\kappa)$, it follows from (24) that the condition

$$
T_{t}=Z_{z}=0
$$

holds unless

$$
\frac{1}{\phi}\left(2 \phi+\kappa \phi_{\kappa}\right)=2+c \quad \frac{1}{\beta}\left(2 \beta+\kappa \beta_{\kappa}\right)=2+c .
$$

These constraints lead us to the special case which we now discuss.

\subsection{Case II: $\phi=\kappa^{c} \Upsilon(\kappa / \tau), \beta=\kappa^{c} \Gamma(\kappa / \tau)$}

Provided that the functions $\phi$ and $\beta$ satisfy (28) (or in the special case $\phi=\phi(\kappa)$ and $\beta=\beta(\kappa)$ satisfy (31)) we can relax the restriction (27). The most general solution to (28) is

$$
\phi=\kappa^{c} \Upsilon\left(\frac{\kappa}{\tau}\right) \quad \beta=\kappa^{c} \Gamma\left(\frac{\kappa}{\tau}\right)
$$

where $\Upsilon(\cdot)$ and $\Gamma(\cdot)$ are arbitrary functions and, as stated earlier, $c$ is an arbitrary constant. For such functions, equations (23) reduce to

$$
U_{u}=V_{v}=Z_{z} \quad T_{t}=Z_{z}(2+c) .
$$

The corresponding symmetry group for (6)-(9) has eight parameters and is

$$
\begin{aligned}
& T=(2+c) \alpha t+t_{0} \\
& \left(\begin{array}{l}
U \\
V \\
Z
\end{array}\right)=\left(\begin{array}{ccc}
\alpha & h & -f \\
-h & \alpha & -g \\
f & g & \alpha
\end{array}\right)\left(\begin{array}{l}
u \\
v \\
z
\end{array}\right)+\left(\begin{array}{l}
u_{0} \\
v_{0} \\
z_{0}
\end{array}\right)
\end{aligned}
$$

where the additional parameter $\alpha$ represents a rescaling. 
2.4. Case III: $\phi=\kappa^{-2 / 3}|\tau|^{-1 / 3}, \beta=0$

The conditions (29) can be relaxed only if $\phi$ and $\beta$ satisfy (30), from which it follows that

$$
|\phi|=M|\kappa|^{-2 / 3}|\tau|^{-1 / 3} \quad \beta=0
$$

where $M>0$ but is otherwise arbitrary. This solution also satisfies (28) with $c=-1$. Scaling the arbitrary constant $M$ out of the equations, leads to the following relation for $\phi$,

$$
\phi=\kappa^{-2 / 3}|\tau|^{-1 / 3} \quad \beta=0
$$

if we require that $\phi$ be positive whatever the sign of $\tau$ (the other possibilities will be evident from (34); $M$ could in fact be taken to have different values for $\tau>0$ and $\tau<0$ ). The corresponding velocity law

$$
\boldsymbol{v}=\kappa^{1 / 3}|\tau|^{-1 / 3} n
$$

has infinite velocity if the torsion of the curve is zero and hence a planar curve propagates with infinite speed. Furthermore, issues of well-posedness remain to be resolved. The corresponding symmetry group has thirteen parameters and takes the from

$$
\begin{aligned}
& T=\frac{a_{11}+a_{22}+a_{33}}{3} t+t_{0} \\
& \left(\begin{array}{l}
U \\
V \\
Z
\end{array}\right)=\left(\begin{array}{lll}
a_{11} & a_{12} & a_{13} \\
a_{21} & a_{22} & a_{23} \\
a_{31} & a_{32} & a_{33}
\end{array}\right)\left(\begin{array}{l}
u \\
v \\
z
\end{array}\right)+\left(\begin{array}{l}
u_{0} \\
v_{0} \\
z_{0}
\end{array}\right) .
\end{aligned}
$$

\section{Global symmetries}

\subsection{Preamble}

We can, in the usual way, use the infinitesimal versions of the group to construct the global forms of transformation under which equations (6)-(9) are invariant. These are given by solving the initial value problem

$$
\begin{array}{ll}
\frac{\partial t^{*}}{\partial \varepsilon}=T\left(t^{*}, u^{*}, v^{*}, w^{*}\right) & \frac{\partial u^{*}}{\partial \varepsilon}=U\left(t^{*}, u^{*}, v^{*}, w^{*}\right) \\
\frac{\partial v^{*}}{\partial \varepsilon}=V\left(t^{*}, u^{*}, v^{*}, w^{*}\right) & \frac{\partial z^{*}}{\partial \varepsilon}=Z\left(t^{*}, u^{*}, v^{*}, w^{*}\right)
\end{array}
$$

with

$$
t^{*}=t \quad u^{*}=u \quad v^{*}=v \quad z^{*}=z \quad \text { on } \quad \varepsilon=0 .
$$

We now solve (39)-(40) for the global groups in the three different cases.

\subsection{Case I: arbitrary $\phi, \beta$}

Before solving (39) it is helpful to make a rotation of the $\left(u^{*}, v^{*}, z^{*}\right)$ coordinates about an appropriate axis to leave (39) in the form

$$
\begin{aligned}
& \frac{\partial t^{*}}{\partial \varepsilon}=t_{0} \\
& \left(\begin{array}{l}
\frac{\partial u^{*}}{\partial \varepsilon} \\
\frac{\partial v^{*}}{\partial \varepsilon} \\
\frac{\partial z^{*}}{\partial \varepsilon}
\end{array}\right)=\left(\begin{array}{ccc}
0 & -\Theta & 0 \\
\Theta & 0 & 0 \\
0 & 0 & 0
\end{array}\right)\left(\begin{array}{l}
u^{*} \\
v^{*} \\
z^{*}
\end{array}\right)+\left(\begin{array}{l}
u_{0} \\
v_{0} \\
z_{0}
\end{array}\right) .
\end{aligned}
$$


Here $t_{0}, u_{0}, v_{0}$ and $z_{0}$ represent translation invariants and $\Theta$ a rotation invariant. The parameter $\Theta$ is related to $f, g$ and $h$ in (26) and the axis of rotation about which the rotation occurs. Integration of (41)-(42) for $\Theta=0$ with respect to $\varepsilon$ yields the obvious translation invariants

$$
t^{*}=t+\varepsilon t_{0} \quad u^{*}=u+\varepsilon u_{0} \quad v^{*}=v+\varepsilon v_{0} \quad z^{*}=z+\varepsilon z_{0} .
$$

For $\Theta \neq 0$ we can set $u_{0}=v_{0}=0$ by translation of $u$ and $v$ and integration of the equations then results in

$$
\begin{array}{ll}
t^{*}=t+\varepsilon t_{0} & u^{*}=\cos (\varepsilon \Theta) u-\sin (\varepsilon \Theta) v \\
v^{*}=\sin (\varepsilon \Theta) u+\cos (\varepsilon \Theta) v & z^{*}=z+\varepsilon z_{0} .
\end{array}
$$

This represents three degrees of freedom $\left(t_{0}, M\right.$ and $\left.z_{0}\right)$ whereas the infinitesimal group (25)-(26) has seven. The difference can be accounted for by noting that an arbitrary rotation and translation of the axis of rotation requires four degrees of freedom. Indeed, we can retrieve the general global group $(\hat{t}, \hat{u}, \hat{v}, \hat{z})$ by making such a rotation and translation to (44) of the form

$$
\hat{t}=t^{*} \quad\left(\begin{array}{c}
\hat{u} \\
\hat{v} \\
\hat{z}
\end{array}\right)=\left(\begin{array}{ccc}
\cos \phi & -\cos \chi \sin \phi & \sin \chi \sin \phi \\
\sin \phi & \cos \chi \cos \phi & -\sin \chi \cos \phi \\
0 & \sin \chi & \cos \chi
\end{array}\right)\left(\begin{array}{c}
u^{*}+u_{0}^{*} \\
v^{*}+v_{0}^{*} \\
z^{*}
\end{array}\right)
$$

where $u_{0}^{*}, v_{0}^{*}, \phi$ and $\chi$ are arbitrary parameters. Thus the Lie group for $(u, v, z)$ is the special Euclidean group $\mathrm{SE}(3)$ which comprises arbitrary rotations and translations of the $(u, v, z)$ space. The global group (44) can also be written in terms of polar coordinates $r, \theta$ and $z$, i.e. in the form

$$
t^{*}=t+\varepsilon t_{0} \quad r^{*}=r \quad \theta^{*}=\theta+\varepsilon \Theta \quad z^{*}=z+\varepsilon z_{0} .
$$

$$
\text { 3.3. Case II: } \phi=\kappa^{c} \Upsilon(\kappa / \tau), \quad \beta=\kappa^{c} \Gamma(\kappa / \tau)
$$

It is again helpful to make a rotation of axes about an appropriate axis. This time we are left with

$$
\begin{aligned}
& \frac{\partial t^{*}}{\partial \varepsilon}=(2+c) \alpha t+t_{0} \\
& \left(\begin{array}{c}
\frac{\partial u^{*}}{\partial \varepsilon} \\
\frac{\partial v^{*}}{\partial \varepsilon} \\
\frac{\partial z^{*}}{\partial \varepsilon}
\end{array}\right)=\left(\begin{array}{ccc}
\alpha & -\Theta & 0 \\
\Theta & \alpha & 0 \\
0 & 0 & \alpha
\end{array}\right)\left(\begin{array}{l}
u^{*} \\
v^{*} \\
z^{*}
\end{array}\right)+\left(\begin{array}{l}
u_{0} \\
v_{0} \\
z_{0}
\end{array}\right)
\end{aligned}
$$

where $\alpha$ is the scaling parameter. Integrating equations (46)-(47) with respect to $\varepsilon$ in the cases $\Theta=\alpha=0$ and $\Theta \neq 0, \alpha=0$ again results in the global transformations (43) and (45) respectively. When $\alpha \neq 0$ integration of these equations gives ( $\operatorname{setting} u_{0}=v_{0}=z_{0}=t_{0}=0$ by translation of $u, v, z$ and $t$ )

$$
\begin{array}{ll}
t^{*}=\exp ((2+c) \alpha \varepsilon) t & u^{*}=\exp (\alpha \varepsilon)(\cos (\varepsilon \Theta) u-\sin (\varepsilon \Theta) v) \\
v^{*}=\exp (\alpha \varepsilon)(\cos (\varepsilon \Theta) u+\cos (\varepsilon \Theta) v) & z^{*}=\exp (\alpha \varepsilon) z .
\end{array}
$$

Writing this in terms of the polar coordinates $r, \theta$ and $z$ we obtain

$t^{*}=\exp ((2+c) \alpha \varepsilon) t \quad r^{*}=\exp (\alpha \varepsilon) r \quad \theta^{*}=\theta+\varepsilon \Theta \quad z^{*}=\exp (\alpha \varepsilon) z$ 
and we see that this transformation is equivalent to a rotation about an arbitrary axis and a rescaling. In the special case $c=-2$ the group (48) is replaced by

$t^{*}=t+\varepsilon t_{0} \quad r^{*}=\exp (\alpha \varepsilon) r \quad \theta^{*}=\theta+\varepsilon \Theta \quad z^{*}=\exp (\alpha \varepsilon) z$.

3.4. Case III: $\phi=\kappa^{-2 / 3}|\tau|^{-1 / 3}, \quad \beta=0$

In this case, we write (39) in the form

$$
\begin{aligned}
& \frac{\partial t^{*}}{\partial \varepsilon}=\frac{1}{3}\left(a_{11}+a_{22}+a_{33}\right) t+t_{0} \\
& \left(\begin{array}{l}
\frac{\partial u^{*}}{\partial \varepsilon} \\
\frac{\partial v^{*}}{\partial \varepsilon} \\
\frac{\partial z^{*}}{\partial \varepsilon}
\end{array}\right)=\underline{\underline{A}}\left(\begin{array}{l}
u^{*} \\
v^{*} \\
z^{*}
\end{array}\right)+\left(\begin{array}{l}
u_{0} \\
v_{0} \\
z_{0}
\end{array}\right) \quad \text { where } \underline{\underline{A}}=\left(\begin{array}{lll}
a_{11} & a_{12} & a_{13} \\
a_{21} & a_{22} & a_{23} \\
a_{31} & a_{32} & a_{33}
\end{array}\right) .
\end{aligned}
$$

Thus the Lie group for $(u, v, z)$ is the general linear group GL(3). We note that the corresponding velocity law (36) has the same curvature dependence as the only twodimensional velocity law with general linear symmetry (see $[16,17])$, namely

$$
\boldsymbol{v}=\kappa^{1 / 3} \boldsymbol{n} .
$$

It is helpful to further subdivide this case into several sub-cases depending upon the type of eigenvalues the matrix $A$ possesses. Although the manipulations which follow are routine, they are worth pursuing in part because we are not aware of any PDEs which have previously been identified as being invariant under GL(3).

3.4.1. Three real distinct eigenvalues. Consider the case where $\underline{A}$ has the three distinct eigenvalues $\lambda_{1}, \lambda_{2}$ and $\lambda_{3}$ with corresponding eigenvectors $\boldsymbol{k}_{1}, \boldsymbol{k}_{2}$ and $\boldsymbol{k}_{3}$. Without loss of generality, we make an appropriate rotation of the $(u, v, z)$ axes to leave $\underline{\underline{A}}, \boldsymbol{k}_{1}, \boldsymbol{k}_{2}$ and $\boldsymbol{k}_{3}$ in the form

$$
\underline{\underline{A}}=\left(\begin{array}{ccc}
\lambda_{1} & a & b \\
0 & \lambda_{2} & c \\
0 & 0 & \lambda_{3}
\end{array}\right) \quad k_{1}=\left(\begin{array}{l}
1 \\
0 \\
0
\end{array}\right) \quad k_{2}=\left(\begin{array}{c}
a \\
\lambda_{2}-\lambda_{1} \\
0
\end{array}\right) \quad k_{3}=\left(\begin{array}{c}
b\left(\lambda_{3}-\lambda_{2}\right)+a c \\
c\left(\lambda_{3}-\lambda_{1}\right) \\
\left(\lambda_{3}-\lambda_{2}\right)\left(\lambda_{3}-\lambda_{1}\right)
\end{array}\right) .
$$

Case IIIa $\left(\lambda_{1} \neq 0, \lambda_{2} \neq 0, \lambda_{3} \neq 0, \lambda_{1}+\lambda_{2}+\lambda_{3} \neq 0\right)$. We can set $u_{0}=v_{0}=z_{0}=t_{0}=0$ by a translation in $u, v, z$ and $t$. The solution to (50)-(51) and (40) is then found to be

$$
\begin{aligned}
t^{*} & =\exp \left(\frac{1}{3}\left(\lambda_{1}+\lambda_{2}+\lambda_{3}\right) \varepsilon\right) t & u^{*} & =J v^{*}+K z^{*}+\mathrm{e}^{\lambda_{1} \varepsilon}(u-J z-K v) \\
v^{*} & =L z^{*}+\mathrm{e}^{\lambda_{2} \varepsilon}(v-L z) & z^{*} & =\mathrm{e}^{\lambda_{3} \varepsilon} z .
\end{aligned}
$$

Here the constants $J, K$ and $L$ depend upon $a, b$ and $c$ and, since $a, b$ and $c$ are arbitrary, are themselves arbitrary.

Case IIIb $\left(\lambda_{1} \neq 0, \lambda_{2} \neq 0, \lambda_{3} \neq 0, \lambda_{1}+\lambda_{2}+\lambda_{3}=0\right)$. Without loss of generality, we can set $u_{0}=v_{0}=z_{0}=0$ by a translation in $u, v$ and $z$. Integrating (50)-(51) and (40) we find that the solution takes the form

$$
\begin{array}{ll}
t^{*}=t+\varepsilon t_{0} & u^{*}=J v^{*}+K z^{*}+\mathrm{e}^{\lambda_{1} \varepsilon}(u-J z-K v) \\
v^{*}=L z^{*}+\mathrm{e}^{\lambda_{2} \varepsilon}(v-L z) & z^{*}=\mathrm{e}^{\lambda_{3} \varepsilon} z .
\end{array}
$$


Here the constants $J, K$ and $L$ again depend upon the arbitrary constants $a, b$ and $c$ and can thus be regarded as arbitrary.

Case IIIC $\left(\lambda_{1} \neq 0, \lambda_{2} \neq 0, \lambda_{3}=0, \lambda_{1}+\lambda_{2}+\lambda_{3} \neq 0\right)$. We can set $u_{0}=v_{0}=t_{0}=0$ by a translation in $u, v$ and $t$. The solution to (50)-(51) and (40) then takes the form

$$
\begin{array}{lll}
t^{*}=\exp \left(\frac{1}{3}\left(\lambda_{1}+\lambda_{2}\right) \varepsilon\right) t & u^{*}=J v^{*}+\mathrm{e}^{\lambda_{1} \varepsilon}(u-J v)+\varepsilon P \\
v^{*}=\mathrm{e}^{\lambda_{2} \varepsilon} v+\varepsilon Q & z^{*}=z+\varepsilon z_{0}
\end{array}
$$

where the constants $J, P$ and $Q$ depend upon the arbitrary constants $a, b, c$ and $z_{0}$ and are thus arbitrary too.

Case IIId $\left(\lambda_{1} \neq 0, \lambda_{2}=-\lambda_{1}, \lambda_{3}=0\right)$. Setting $u_{0}=v_{0}=t_{0}=0$ by a translation in $u, v$ and $t$ we find that the solution to (50)-(51) and (40) takes the form

$$
\begin{array}{ll}
t^{*}=t+\varepsilon t_{0} & u^{*}=J v^{*}+\mathrm{e}^{\lambda_{1} \varepsilon}(u-J v)+\varepsilon P \\
v^{*}=\mathrm{e}^{-\lambda_{1} \varepsilon} v+\varepsilon Q & z^{*}=z+\varepsilon z_{0}
\end{array}
$$

where $J, P$ and $Q$ depend upon the arbitrary constants $a, b, c$ and $z_{0}$ and are hence arbitrary also.

3.4.2. One real and two complex eigenvalues. Consider the case where $\underline{\underline{A}}$ has one real eigenvector $\lambda$ and two complex eigenvectors $\Lambda+\mathrm{i} \omega$ and $\Lambda-\mathrm{i} \omega$ with corresponding eigenvectors $\boldsymbol{k}, \boldsymbol{l}+\mathrm{i} \boldsymbol{m}, \boldsymbol{l}-\mathrm{i} \boldsymbol{m}$ respectively (here $\Lambda, \omega, \boldsymbol{l}$ and $\boldsymbol{m}$ are all real). Without loss of generality, we may make a rotation of the coordinate axes so that $\boldsymbol{l}$ and $\boldsymbol{m}$ lie in the plane $z=0$ and take the form $(1,0,0)^{T}$ and $\left(m_{1}, m_{2}, 0\right)^{T}$ respectively, and $k=\left(k_{1}, k_{2}, 1\right)^{T}$. In these new coordinates, the matrix $\underline{\underline{A}}$ has the form

$$
\underline{\underline{A}}=\left(\begin{array}{lll}
a & b & e \\
c & d & f \\
0 & 0 & \lambda
\end{array}\right) .
$$

Case IIIe $(\lambda+2 \Lambda \neq 0, \lambda \neq 0)$. In this case, where we set $u_{0}=v_{0}=z_{0}=t_{0}=0$ by a translation in $u, v, z$ and $t$, the general solution to (50)-(51) is of the form

$$
\begin{aligned}
& t^{*}=\exp \left(\frac{1}{3}(\lambda+2 \Lambda) \varepsilon\right) t \\
& \left(\begin{array}{l}
u^{*} \\
v^{*} \\
z^{*}
\end{array}\right)=D \exp (\Lambda \varepsilon)(\cos (\omega \varepsilon+\alpha) \boldsymbol{m}+\sin (\omega \varepsilon+\alpha) \boldsymbol{l})+C \exp (\lambda \varepsilon) \boldsymbol{k}
\end{aligned}
$$

where $C, D$ and $\alpha$ are arbitrary constants. The solution to (50)-(51) and (40) thus takes the form

$$
\begin{aligned}
t^{*} & =\exp \left(\frac{1}{3}(\lambda+2 \Lambda) \varepsilon\right) t \\
u^{*} & =\exp (\Lambda \varepsilon) \cos (\omega \varepsilon+\alpha)\left(\frac{u-J v-K z}{\cos \alpha}\right)+J v^{*}+K z^{*} \\
v^{*} & =\exp (\Lambda \varepsilon) \sin (\omega \varepsilon+\alpha) \frac{v-L z}{\sin \alpha}+L z^{*} \\
z^{*} & =\exp (\lambda \varepsilon) z
\end{aligned}
$$

where $J, K$ and $L$ depend upon the arbitrary constants $k_{1}, k_{2}, m_{1}$ and $m_{2}$ and are thus themselves arbitrary.

Case IIIf $(\lambda+2 \Lambda=0, \lambda \neq 0)$. Setting $u_{0}=v_{0}=z_{0}=0$ by translations in $u, v$, and $z$, the solution to (50)-(51) and (40) takes the form 


$$
\begin{aligned}
& t^{*}=t+\varepsilon t_{0} \\
& u^{*}=\exp (\Lambda \varepsilon) \cos (\omega \varepsilon+\alpha)\left(\frac{u-J v-K z}{\cos \alpha}\right)+J v^{*}+K z^{*} \\
& v^{*}=\exp (\Lambda \varepsilon) \sin (\omega \varepsilon+\alpha) \frac{v-L z}{\sin \alpha}+L z^{*} \\
& z^{*}=\exp (\lambda \varepsilon) z
\end{aligned}
$$

where $J, K$ and $L$ depend upon the arbitrary constants $k_{1}, k_{2}, m_{1}$ and $m_{2}$ and are thus themselves arbitrary.

Case IIIg $(\lambda+2 \Lambda \neq 0, \lambda=0)$. Setting $u_{0}=v_{0}=t_{0}=0$ by a translation in $u, v$, and $t$, we find that the solution to (50)-(51) and (40) has the form

$$
\begin{aligned}
t^{*} & =\exp \left(\frac{1}{3}(\lambda+2 \Lambda) \varepsilon\right) t \\
u^{*} & =\exp (\Lambda \varepsilon) \cos (\omega \varepsilon+\alpha)\left(\frac{u-J v-K z}{\cos \alpha}\right)+J v^{*}+K z^{*} \\
v^{*} & =\exp (\Lambda \varepsilon) \sin (\omega \varepsilon+\alpha) \frac{v-L z}{\sin \alpha}+L z^{*} \\
z^{*} & =z+\varepsilon z_{0}
\end{aligned}
$$

where $z_{0}$ is arbitrary and $J, K$ and $L$, which depend upon the arbitrary constants $k_{1}, k_{2}, m_{1}$ and $m_{2}$, are again arbitrary.

Case IIIh $(\lambda=\Lambda=0)$. Setting $u_{0}=v_{0}=0$ by a translation in $u$ and $v$, we find that the solution to (50)-(51) and (40) has the form

$$
\begin{aligned}
t^{*} & =t+\varepsilon t_{0} \\
u^{*} & =\cos (\omega \varepsilon+\alpha)\left(\frac{u-J v-K z}{\cos \alpha}\right)+J v^{*}+K z^{*} \\
v^{*} & =\sin (\omega \varepsilon+\alpha) \frac{v-L z}{\sin \alpha}+L z^{*} \\
z^{*} & =z+\varepsilon z_{0}
\end{aligned}
$$

where $z_{0}, t_{0}$ are arbitrary, as are $J, K$ and $L$, which depend upon the arbitrary constants $k_{1}, k_{2}, m_{1}$ and $m_{2}$.

3.4.3. One distinct and two identical real eigenvalues. Consider the case where $\underline{A}$ has a repeated eigenvalue $\Lambda$ with a repeated eigenvector $k$ (the case where $k$ is not repeated is encompassed by the results of section 3.4.1). Without loss of generality, we may make two rotations to leave $\underline{A}$ and $k$ in the forms

$$
\underline{\underline{A}}=\left(\begin{array}{ccc}
\Lambda & a & b \\
0 & \Lambda & c \\
0 & 0 & \lambda
\end{array}\right) \quad k=\left(\begin{array}{l}
1 \\
0 \\
0
\end{array}\right)
$$

where $a, b$ and $c$ are arbitrary constants.

Case IIIi $(2 \Lambda+\lambda \neq 0, \lambda \neq 0$ and $\Lambda \neq 0)$. Setting $u_{0}=v_{0}=z_{0}=t_{0}=0$ by a translation in $u, v, z$ and $t$ we find the general solution to (50)-(51) and (40) to be 


$$
\begin{aligned}
& t^{*}=\exp \left(\frac{1}{3}(\lambda+2 \Lambda) \varepsilon\right) t \\
& \left(\begin{array}{l}
u^{*} \\
v^{*} \\
z^{*}
\end{array}\right)=B \mathrm{e}^{\Lambda \varepsilon}\left(\begin{array}{l}
1 \\
0 \\
0
\end{array}\right)+C \mathrm{e}^{\lambda \varepsilon}\left(\begin{array}{c}
b(\lambda-\Lambda)+a c \\
c(\lambda-\Lambda) \\
(\lambda-\Lambda)^{2}
\end{array}\right)+D \mathrm{e}^{\Lambda \varepsilon}\left(\begin{array}{c}
a \varepsilon \\
1 \\
0
\end{array}\right)
\end{aligned}
$$

where the quantities $B, C$ and $D$ are determined by the initial condition (40). Since the three parameters $a, b$ and $c$ are, in effect, arbitrary the upshot is that we can choose to write the results for $u^{*}, v^{*}$ and $z^{*}$ in the form

$$
u^{*}=\mathrm{e}^{\Lambda \varepsilon}(u-K z)+K z^{*}+\varepsilon P\left(v^{*}-L z^{*}\right) \quad v^{*}=L z^{*}+\mathrm{e}^{\Lambda \varepsilon}(v-L z) \quad z^{*}=\mathrm{e}^{\lambda \varepsilon} z
$$

where $P, K$ and $L$ are arbitrary constants.

Case IIIj $(2 \Lambda+\lambda=0$ and $\lambda \neq 0)$. Setting $u_{0}=v_{0}=z_{0}=0$ by a translation in $u, v$ and $z$ and solving (50)-(51) and (40) results in a solution of the form

$t^{*}=t+\varepsilon t_{0} \quad u^{*}=\mathrm{e}^{\Lambda \varepsilon}(u-K z)+K z^{*}+\varepsilon P\left(v^{*}-L z^{*}\right)$

$v^{*}=L z^{*}+\mathrm{e}^{\Lambda \varepsilon}(v-L z) \quad z^{*}=\mathrm{e}^{\lambda \varepsilon} z$

where $t_{0}$ is arbitrary and $K, L$ and $P$ depend upon the arbitrary parameters $a, b$ and $c$ and so are arbitrary themselves.

Case IIIk $(\lambda=0$ and $\Lambda \neq 0)$. Setting $u_{0}=v_{0}=t_{0}=0$ by a translation in $u, v$ and $t$ and solving (50)-(51) and (40) results in a solution of the form

$$
\begin{array}{ll}
t^{*}=\exp \left(\frac{2}{3} \Lambda \varepsilon\right) t & u^{*}=\mathrm{e}^{\Lambda \varepsilon}(u+\varepsilon P v)+\varepsilon Q \\
v^{*}=\mathrm{e}^{\Lambda \varepsilon} v+\varepsilon M & z^{*}=z+\varepsilon z_{0}
\end{array}
$$

where $z_{0}$ is arbitrary and $P, Q$ and $M$ depend upon the arbitrary parameters $a, b$ and $c$ and so are arbitrary themselves.

Case IIIl $(\lambda \neq 0$ and $\Lambda=0)$. Setting $t_{0}=z_{0}=0$ by a translation in $t$ and $z$ and solving (50)-(51) and (40) we find

$t^{*}=\exp \left(\frac{1}{3} \lambda \varepsilon\right) t$

$u^{*}=\left(u-\left(\frac{a c}{\lambda^{2}}+\frac{b}{\lambda}\right) z\right)+\mathrm{e}^{\lambda \varepsilon}\left(\frac{a c}{\lambda^{2}}+\frac{b}{\lambda}\right) z+\varepsilon\left(u_{0}+a\left(v-\frac{c}{\lambda} z\right)\right)+\varepsilon^{2} \frac{a v_{0}}{2}$

$v^{*}=\left(v-\frac{c}{\lambda} z\right)+\mathrm{e}^{\lambda \varepsilon} \frac{c}{\lambda} z+\varepsilon v_{0}$

$z^{*}=\mathrm{e}^{\lambda \varepsilon} z$.

where $u_{0}, v_{0}, a, b$ and $c$ are all arbitrary.

3.4.4. Three identical real eigenvalues. Consider the case where $\underline{\underline{A}}$ has the eigenvalue $\Lambda$ repeated three times each with eigenvector $k$ (the case where $k$ is not repeated is covered in section 3.4.1 and the case where it is repeated only twice is covered in section 3.4.3). We may again make two rotations to leave $\underline{\underline{A}}$ and $k$ in the following convenient forms:

$$
\underline{\underline{A}}=\left(\begin{array}{ccc}
\Lambda & a & b \\
0 & \Lambda & c \\
0 & 0 & \Lambda
\end{array}\right) \quad k=\left(\begin{array}{l}
1 \\
0 \\
0
\end{array}\right) \text {. }
$$


Case IIIm $(\Lambda \neq 0)$. Setting $u_{0}=v_{0}=z_{0}=t_{0}=0$ by a translation in $u, v, z$ and $t$ and solving (50)-(51) and (40) results in solution

$$
\begin{aligned}
t^{*} & =\exp (\Lambda \varepsilon) t & u^{*} & =\mathrm{e}^{\Lambda \varepsilon} u+\varepsilon \mathrm{e}^{\Lambda \varepsilon}(a v+b z)+\varepsilon^{2} \mathrm{e}^{\Lambda \varepsilon} \frac{a c}{2} z \\
v^{*} & =\mathrm{e}^{\Lambda \varepsilon} v+\varepsilon \mathrm{e}^{\Lambda \varepsilon} c z & z^{*} & =\mathrm{e}^{\Lambda \varepsilon} z
\end{aligned}
$$

where $a, b$ and $c$ are arbitrary.

Case IIIn $(\Lambda=0)$. In this case, the solution to (50)-(51) and (40) has the form

$$
\begin{aligned}
& t^{*}=t+\varepsilon t_{0} \\
& u^{*}=u+\varepsilon\left(a v+b z+u_{0}\right)+\frac{\varepsilon^{2}}{2}\left(a\left(c z+v_{0}\right)+b z_{0}\right)+\varepsilon^{3} \frac{a c z_{0}}{6} \\
& v^{*}=v+\varepsilon\left(v_{0}+c z\right)+\varepsilon^{2} \frac{c z_{0}}{2} \\
& z^{*}=z+\varepsilon z_{0}
\end{aligned}
$$

where the constants $\left(t_{0}, u_{0}, v_{0}, z_{0}, a, b, c\right)$ are all arbitrary.

\section{Similarity reductions}

\subsection{Case I: $\phi$ and $\beta$ arbitrary functions of $\kappa$ and $\tau$}

Case Ia $(M=0)$. The invariants (i.e. quantities that are not functions of $\varepsilon$ ) of the global transformation (43) are, when we set $u_{0}=v_{0}=0$,

$$
\begin{array}{lll}
u & v & z
\end{array}
$$

where $q=z_{0} / t_{0}$. This leads to the travelling wave reduction to (6)-(9) of the form

$$
u=U(z-q t) \quad v=V(z-q t) .
$$

Case Ib $(M \neq 0)$. Invariants of the global transformation (45) are

$$
\theta-c t \quad r=\left(u^{2}+v^{2}\right)^{1 / 2} \quad z-q t
$$

where $c=M / t_{0}$ or, when $t_{0}=0$,

$$
z-k \theta \quad r=\left(u^{2}+v^{2}\right)^{1 / 2} \quad t
$$

where $k=z_{0} / M$. In the former case, this leads to a travelling rotating wave reduction

$$
r=R(\theta-c t) \quad z=q t+f(\theta-c t)
$$

where the arbitrary constants $q$ and $c$ are respectively the velocity and angular velocity of the wave. The latter case results in the helical similarity reduction

$$
r=R(t) \quad z=k \theta+f(t) .
$$

\subsection{Case II: $\phi=\kappa^{c} \Upsilon(\kappa / \tau), \beta=\kappa^{c} \Gamma(\kappa / \tau)$}

We note first that the special case $c=0, \Upsilon=1, \Gamma=0$ has already been addressed in [13]. In general where functions $\phi$ and $\beta$ are of the above form with $c \neq-2$, equations (6)-(9) are invariant under the transformation (48) for which the invariants are

$$
\frac{r^{2}}{t^{2 /(2+c)}}=\frac{\left(u^{2}+v^{2}\right)}{t^{2 /(2+c)}} \quad \frac{z}{t^{1 /(2+c)}} \quad \theta-\frac{M}{(2+c) \alpha} \log |t| .
$$


This implies the possibility of additional similarity reductions of the form

$$
r=t^{1 /(2+c)} R(\theta-p \log t) \quad z=t^{1 /(2+c)} f(\theta-p \log t)
$$

and

$r=(-t)^{1 /(2+c)} R(\theta-p \log (-t)) \quad z=(-t)^{1 /(2+c)} f(\theta-p \log (-t))$.

In the special case $c=-2$ invariants of (49), with $t_{0} \neq 0$, are

$$
\theta-\frac{\Theta}{t_{0}} t \quad r \exp \left(-\frac{\alpha}{t_{0}} t\right) \quad z \exp \left(-\frac{\alpha}{t_{0}} t\right)
$$

so we have the similarity reduction

$$
r=\exp (\beta t) R(\theta-c t) \quad z=\exp (\beta t) f(\theta-c t) .
$$

When $t_{0}=0$, the invariants of (49) are

$$
t \quad z \exp \left(\frac{\alpha}{\Theta} \theta\right) \quad r \exp \left(\frac{\alpha}{\Theta} \theta\right)
$$

which implies that there is a similarity reduction of the form

$$
r=\exp (M \theta) R(t) \quad z=\exp (M \theta) f(t) .
$$

4.3. Case III: $\phi=\kappa^{-2 / 3}|\tau|^{-1 / 3}, \quad \beta=0$.

When $\phi=\kappa^{-2 / 3}|\tau|^{-1 / 3}$ and $\beta=0$ equations (6)-(9) reduce to the significantly simpler forms

$$
\begin{aligned}
& u_{t}=\frac{u_{z z}}{\left|u_{z z} v_{z z z}-u_{z z z} v_{z z}\right|^{1 / 3}} \\
& v_{t}=\frac{v_{z z}}{\left|u_{z z} v_{z z z}-u_{z z z} v_{z z}\right|^{1 / 3}} .
\end{aligned}
$$

\subsubsection{Three real distinct eigenvalues $\lambda_{1}, \lambda_{2}$ and $\lambda_{3}$}

Case IIIa. Invariants of the global transformation (52) are

$$
z t^{-3 \lambda_{3} /\left(\lambda_{1}+\lambda_{2}+\lambda_{3}\right)} \quad(u-J v-K z) t^{-3 \lambda_{1} /\left(\lambda_{1}+\lambda_{2}+\lambda_{3}\right)} \quad(v-L z) t^{-3 \lambda_{2} /\left(\lambda_{1}+\lambda_{2}+\lambda_{3}\right)} .
$$

We hence look for a similarity reductions to (6)-(9) of the form

$u=J z+K v+( \pm t)^{\alpha} F\left(z( \pm t)^{-\beta}\right) \quad v=L z+( \pm t)^{3-\alpha-\beta} G\left(z( \pm t)^{-\beta}\right)$

where $\alpha=3 \lambda_{1} /\left(\lambda_{1}+\lambda_{2}+\lambda_{3}\right)$ and $\beta=3 \lambda_{3} /\left(\lambda_{1}+\lambda_{2}+\lambda_{3}\right)$ and are hence arbitrary (since $\lambda_{1}, \lambda_{2}$ and $\lambda_{3}$ are arbitrary), as are $J, K$ and $L$. Substituting (91) into (89)-(90) gives

$$
\begin{aligned}
& \pm\left(\alpha F-\beta \eta F^{\prime}\right)=\frac{F^{\prime \prime}}{\left|G^{\prime \prime \prime} F^{\prime \prime}-G^{\prime \prime} F^{\prime \prime \prime}\right|^{1 / 3}} \\
& \pm\left((3-\alpha-\beta) G-\beta \eta G^{\prime}\right)=\frac{G^{\prime \prime}}{\left|G^{\prime \prime \prime} F^{\prime \prime}-G^{\prime \prime} F^{\prime \prime \prime}\right|^{1 / 3}}
\end{aligned}
$$

Case IIIb. Invariants of the global transformation (52) are

$z \exp \left(-\lambda_{3} t / t_{0}\right) \quad(v-L z) \exp \left(-\lambda_{2} t / t_{0}\right) \quad(u-J v-K z) \exp \left(-\lambda_{1} t / t_{0}\right)$ 
which suggests the possibility of similarity transforms of the form

$$
\begin{aligned}
& u=J v+K z+\exp (\beta t) F(z \exp (-\alpha t)) \\
& v=L z+\exp (-(\alpha+\beta) t) G(z \exp (-\alpha t))
\end{aligned}
$$

where $\alpha=\lambda_{3} / t_{0}$ and $\beta=\lambda_{1} / t_{0}$ may be regarded as arbitrary. Substituting the above into (89)-(90) yields

$-(\alpha+\beta) G-\alpha \eta G^{\prime}=\frac{G^{\prime \prime}}{\left|G^{\prime \prime \prime} F^{\prime \prime}-G^{\prime \prime} F^{\prime \prime \prime}\right|^{1 / 3}} \quad \beta F-\alpha \eta F^{\prime}=\frac{F^{\prime \prime}}{\left|G^{\prime \prime \prime} F^{\prime \prime}-G^{\prime \prime} F^{\prime \prime \prime}\right|^{1 / 3}}$.

In the case $t_{0}=0$ the invariants of the global transformation (52) are

$$
t \quad(v-L z) z^{-\lambda_{2} / \lambda_{3}} \quad(u-J v-K z) z^{-\lambda_{1} / \lambda_{3}}
$$

and hence we look for similarity solutions of the form

$$
\begin{aligned}
& u=J v+K z+( \pm z)^{\alpha} F(t) \\
& v=L z+( \pm z)^{-(1+\alpha)} G(t)
\end{aligned}
$$

where $\alpha=\lambda_{1} / \lambda_{3}$ and is thus arbitrary. In this case, equations (89)-(90) reduce to

$$
\begin{aligned}
F^{\prime} & =\frac{\alpha(\alpha-1) F}{|\alpha(\alpha-1)(1+\alpha)(\alpha+2)(2 \alpha+1) F G|^{1 / 3}} \\
G^{\prime} & =\frac{(\alpha+1)(\alpha+2) G}{|\alpha(\alpha-1)(1+\alpha)(\alpha+2)(2 \alpha+1) F G|^{1 / 3}} .
\end{aligned}
$$

Case IIIc. In this case, the invariants of the global transformation cannot easily be found, so we use the invariant surface condition to determine the form of similarity solution in the usual way (see, for example, [6]) from the infinitesimal. This gives solutions of the form

$$
u=J v+K z+( \pm t)^{\alpha} F(z-M \log ( \pm t)) \quad v=L z+( \pm t)^{3-\alpha} G(z-M \log ( \pm t))
$$

where $\alpha, J, K$ and $L$ are arbitrary constants. By substituting (96) into (89)-(90) we find

$\pm\left\{\alpha F-M F^{\prime}\right\}=\frac{F^{\prime \prime}}{\left|G^{\prime \prime \prime} F^{\prime \prime}-G^{\prime \prime} F^{\prime \prime \prime}\right|^{1 / 3}} \quad \pm\left\{(3-\alpha) G-M G^{\prime}\right\}=\frac{G^{\prime \prime}}{\left|G^{\prime \prime \prime} F^{\prime \prime}-G^{\prime \prime} F^{\prime \prime \prime}\right|^{1 / 3}}$.

Case IIId. Here it is again nontrivial to find the invariants of the global transformation and so we resort to the invariant surface condition to give either

$$
u=J v+K z+\mathrm{e}^{\alpha t} F(z-c t) \quad v=L z+\mathrm{e}^{-\alpha t} G(z-c t)
$$

or

$$
u=J v+K z+\mathrm{e}^{\alpha z} F(t) \quad v=L z+\mathrm{e}^{-\alpha z} G(t)
$$

where $\alpha, c, J, K$ and $L$ are arbitrary constants. Substituting (97) into (89)-(90) gives

$\alpha F-c F^{\prime}=\frac{F^{\prime \prime}}{\left|G^{\prime \prime \prime} F^{\prime \prime}-G^{\prime \prime} F^{\prime \prime \prime}\right|^{1 / 3}} \quad-\alpha G-c G^{\prime}=\frac{G^{\prime \prime}}{\left|G^{\prime \prime \prime} F^{\prime \prime}-G^{\prime \prime} F^{\prime \prime \prime}\right|^{1 / 3}}$.

Substituting (98) into (89)-(90) gives

$$
F^{\prime}=\frac{\alpha^{2} F}{2\left|\alpha^{5} G F\right|^{1 / 3}} \quad G^{\prime}=\frac{\alpha^{2} G}{2\left|\alpha^{5} G F\right|^{1 / 3}} .
$$




\subsubsection{One real and two complex eigenvalues $\lambda, \Lambda+\mathrm{i} \omega, \Lambda-\mathrm{i} \omega$}

Case IIIe. Once again it is necessary to use the invariant surface condition to find the similarity reductions. We find two similarity reductions of the form

$$
\begin{aligned}
& u=J v+K z+( \pm t)^{3 / 2-\beta / 2} \rho\left(z( \pm t)^{-\beta}\right) \cos \left(M \log ( \pm t)-\phi\left(z( \pm t)^{-\beta}\right)\right) \\
& v=L z+q( \pm t)^{3 / 2-\beta / 2} \rho\left(z( \pm t)^{-\beta}\right) \sin \left(M \log ( \pm t)-\phi\left(z( \pm t)^{-\beta}\right)\right)
\end{aligned}
$$

Here $\beta, J, K, L$ and $q$ are arbitrary constants. Substituting (99) into (89)-(90) we find

$\pm\left\{\left(\frac{3}{2}-\frac{\beta}{2}\right) \rho-\beta \eta \rho^{\prime}\right\}=\frac{\rho^{\prime \prime}-\rho \phi^{\prime 2}}{|q \Upsilon(\eta)|^{1 / 3}} \quad \pm\left\{M \rho+\beta \eta \rho \phi^{\prime}\right\}=-\frac{2 \rho^{\prime} \phi^{\prime}+\rho \phi^{\prime \prime}}{|q \Upsilon(\eta)|^{1 / 3}}$

where

$$
\begin{gathered}
\Upsilon(\eta)=\left(\rho^{\prime \prime}-\rho \phi^{\prime 2}\right)^{\prime}\left(2 \rho^{\prime} \phi^{\prime}+\rho \phi^{\prime \prime}\right)-\left(2 \rho^{\prime} \phi^{\prime}+\rho \phi^{\prime \prime}\right)^{\prime}\left(\rho^{\prime \prime}-\rho \phi^{\prime 2}\right) \\
-\phi^{\prime}\left[\left(\rho^{\prime \prime}-\rho \phi^{\prime 2}\right)^{2}+\left(2 \rho^{\prime} \phi^{\prime}+\rho \phi^{\prime \prime}\right)^{2}\right] .
\end{gathered}
$$

Case IIIf. As before, we use the invariant surface condition to determine the similarity reduction; we find that it either takes the form

$$
\begin{aligned}
& u=J v+K z+\mathrm{e}^{-\beta t} \rho\left(z \mathrm{e}^{-2 \beta t}\right) \cos \left(M t-\phi\left(z \mathrm{e}^{-2 \beta t}\right)\right) \\
& v=L z+q \mathrm{e}^{-\beta t} \rho\left(z \mathrm{e}^{-2 \beta t}\right) \sin \left(M t-\phi\left(z \mathrm{e}^{-2 \beta t}\right)\right)
\end{aligned}
$$

or

$$
\begin{aligned}
& u=J v+K z+( \pm z)^{-1 / 2} \rho(t) \cos (M \log ( \pm z)-\phi(t)) \\
& v=L z+q( \pm z)^{-1 / 2} \rho(t) \sin (M \log ( \pm z)-\phi(t))
\end{aligned}
$$

where $\beta, J, K, L$ and $M$ are all arbitrary. In the former case, we find that $\rho$ and $\phi$ obey the equations

$$
M \rho+2 \beta \eta \rho \phi^{\prime}=-\frac{2 \rho^{\prime} \phi^{\prime}+\rho \phi^{\prime \prime}}{|q \Upsilon(\eta)|^{1 / 3}} \quad-\left(\beta \rho+2 \beta \eta \rho^{\prime}\right)=\frac{\rho^{\prime \prime}-\rho \phi^{\prime 2}}{|q \Upsilon(\eta)|^{1 / 3}}
$$

while in the latter they obey the equations

$\rho^{\prime}=\frac{\left(\frac{3}{4}-M^{2}\right) \rho}{\left|q M\left\{\left(\frac{3}{4}+M^{2}\right)^{2}+M^{2}\right\} \rho^{2}\right|^{1 / 3}} \quad \rho \phi^{\prime}=\frac{2 M \rho}{\left|q M\left\{\left(\frac{3}{4}+M^{2}\right)^{2}+M^{2}\right\} \rho^{2}\right|^{1 / 3}}$.

Here $\Upsilon$ is given by (100).

Case IIIg. The similarity reduction takes the form

$u=J v+K z+( \pm t)^{3 / 2} \rho(z-M \log ( \pm t)) \cos (\Omega \log ( \pm t)-\phi(z-M \log ( \pm t)))$

$v=L z+q( \pm t)^{3 / 2} \rho(z-M \log ( \pm t)) \sin (\Omega \log ( \pm t)-\phi(z-M \log ( \pm t)))$

where $\Omega, J, K, L$ and $M$ are all arbitrary. Substituting the above into (89)-(90) gives

$$
\pm\left(\frac{3}{2} \rho-M \rho^{\prime}\right)=\frac{\rho^{\prime \prime}-\rho \phi^{\prime 2}}{|q \Upsilon(\eta)|^{1 / 3}} \quad \pm\left(\Omega \rho+M \rho \phi^{\prime}\right)=-\frac{2 \rho^{\prime} \phi^{\prime}+\rho \phi^{\prime \prime}}{|q \Upsilon(\eta)|^{1 / 3}}
$$

where $\Upsilon$ is given by (100).

Case IIIh. The similarity reduction takes either the form

$$
\begin{aligned}
& u=J v+K z+\rho(z-c t) \cos (\Omega t-\phi(z-c t)) \\
& v=L z+q \rho(z-c t) \sin (\Omega t-\phi(z-c t))
\end{aligned}
$$


or

$u=J v+K z+\rho(t) \cos (\Omega z-\phi(t)) \quad v=L z+q \rho(t) \sin (\Omega z-\phi(t))$

where $\Omega, c, J, K$ and $L$ are all arbitrary. In the former case, we find the following equations for $\rho$ and $\phi$,

$$
\rho\left(\Omega+c \phi^{\prime}\right)=-\frac{2 \rho^{\prime} \phi^{\prime}+\rho \phi^{\prime \prime}}{|q \Upsilon(\eta)|^{1 / 3}} \quad-c \rho^{\prime}=\frac{\rho^{\prime \prime}-\rho \phi^{\prime 2}}{|q \Upsilon(\eta)|^{1 / 3}}
$$

(where $\Upsilon$ is given by (100)) while in the latter we obtain

$$
\rho^{\prime}=-\frac{\Omega^{2} \rho}{\left|q \Omega^{5} \rho^{2}\right|^{1 / 3}} \quad \rho \phi^{\prime}=0
$$

\subsubsection{One distinct and two identical real eigenvalues $\lambda$ and $\Lambda$}

Case IIII. The similarity reduction takes the form

$$
\begin{aligned}
& u=K z+( \pm t)^{(3 / 2-\beta / 2)}\left[F\left(z( \pm t)^{-\beta}\right)+p \log ( \pm t) G\left(z( \pm t)^{-\beta}\right)\right] \\
& v=L z+( \pm t)^{(3 / 2-\beta / 2)} G\left(z( \pm t)^{-\beta}\right)
\end{aligned}
$$

where $p, \beta, K$ and $L$ are all arbitrary. Substituting (106) into (89)-(90) gives the following equations for $F$ and $G$ :

$$
\begin{aligned}
& \pm\left(p G+\left(\frac{3}{2}-\frac{\beta}{2}\right) F-\beta \eta F^{\prime}\right)=\frac{F^{\prime \prime}}{\left|G^{\prime \prime \prime} F^{\prime \prime}-G^{\prime \prime} F^{\prime \prime \prime}\right|^{1 / 3}} \\
& \pm\left(\left(\frac{3}{2}-\frac{\beta}{2}\right) G-\beta \eta G^{\prime}\right)=\frac{G^{\prime \prime}}{\left|G^{\prime \prime \prime} F^{\prime \prime}-G^{\prime \prime} F^{\prime \prime \prime}\right|^{1 / 3}}
\end{aligned}
$$

Case IIIj. The similarity reductions take either the form

$u=K z+\mathrm{e}^{\alpha t} F\left(z \mathrm{e}^{2 \alpha t}\right)+p t \mathrm{e}^{\alpha t} G\left(z \mathrm{e}^{2 \alpha t}\right) \quad v=L z+\mathrm{e}^{\alpha t} G\left(z \mathrm{e}^{2 \alpha t}\right)$.

or

$$
u=K z+( \pm z)^{-1 / 2} F(t)+p( \pm z)^{-1 / 2} \log ( \pm z) G(t) \quad v=L z+( \pm z)^{-1 / 2} G(t)
$$

where $K, L, p$ and $\alpha$ are arbitrary. Making the substitution (107) into (89)-(90) gives

$p G+\alpha F+2 \alpha \eta F^{\prime}=\frac{F^{\prime \prime}}{\left|G^{\prime \prime \prime} F^{\prime \prime}-G^{\prime \prime} F^{\prime \prime \prime}\right|^{1 / 3}} \quad \alpha G+2 \alpha \eta G^{\prime}=\frac{G^{\prime \prime}}{\left|G^{\prime \prime \prime} F^{\prime \prime}-G^{\prime \prime} F^{\prime \prime \prime}\right|^{1 / 3}}$

whereas for the ansatz (108) we obtain

$$
F^{\prime}=\frac{3 F / 4-2 p G}{\left|9 p G^{2} / 16\right|^{1 / 3}} \quad G^{\prime}=\frac{3 F / 4}{\left|9 p G^{2} / 16\right|^{1 / 3}} .
$$

Case IIIk. The similarity reductions are

$$
\begin{aligned}
& u=K z+( \pm t)^{3 / 2}[F(z-M \log ( \pm t))+p \log ( \pm t) G(z-M \log ( \pm t))] \\
& v=L z+( \pm t)^{3 / 2} G(z-M \log ( \pm t))
\end{aligned}
$$

where $M, K$ and $L$ are all arbitrary. Substituting (109) into (89)-(90) yields

$$
\begin{aligned}
& \pm\left(\frac{3}{2} F-M F^{\prime}+p G\right)=\frac{F^{\prime \prime}}{\left|G^{\prime \prime \prime} F^{\prime \prime}-G^{\prime \prime} F^{\prime \prime \prime}\right|^{1 / 3}} \\
& \pm\left(\frac{3}{2} G-M G^{\prime}\right)=\frac{G^{\prime \prime}}{\left|G^{\prime \prime \prime} F^{\prime \prime}-G^{\prime \prime} F^{\prime \prime \prime}\right|^{1 / 3}} .
\end{aligned}
$$


Case IIIl. The similarity reductions are

$$
\begin{aligned}
& u=J v+K z-\frac{p q}{2}(\log ( \pm t))^{2}+p(v-r z) \log ( \pm t)+F\left(z( \pm t)^{-3}\right) \\
& v=L z+q \log ( \pm t)+G\left(z( \pm t)^{-3}\right)
\end{aligned}
$$

where $p, q, J, K$ and $L$ are all arbitrary. Substituting (110) into (89)-(90) gives

$$
\begin{aligned}
& \pm\left(J q-3 \eta J G^{\prime}+p G-3 \eta F^{\prime}\right)=\frac{F^{\prime \prime}+J G^{\prime \prime}}{\left|F^{\prime \prime} G^{\prime \prime \prime}-G^{\prime \prime} F^{\prime \prime \prime}\right|^{1 / 3}} \\
& \pm\left(q-3 \eta G^{\prime}\right)=\frac{G^{\prime \prime}}{\left|F^{\prime \prime} G^{\prime \prime \prime}-G^{\prime \prime} F^{\prime \prime \prime}\right|^{1 / 3}} .
\end{aligned}
$$

\subsubsection{Three identical real eigenvalues $\Lambda$}

Case IIIm. The similarity reductions are

$u=K z+\frac{p q}{2} z(\log ( \pm t))^{2}+( \pm t) F\left(\frac{z}{( \pm t)}\right)+p( \pm t) \log ( \pm t) G\left(\frac{z}{( \pm t)}\right)$

$v=L z+q z \log ( \pm t)+( \pm t) G\left(\frac{z}{( \pm t)}\right)$

where $p, q, K$ and $L$ are all arbitrary. Substitution of (111) into (89)-(90) gives

$\pm\left(F-\eta F^{\prime}+p G\right)=\frac{F^{\prime \prime}}{\left|G^{\prime \prime \prime} F^{\prime \prime}-G^{\prime \prime} F^{\prime \prime \prime}\right|^{1 / 3}} \quad \pm\left(G-\eta G^{\prime}+q \eta\right)=\frac{G^{\prime \prime}}{\left|G^{\prime \prime \prime} F^{\prime \prime}-G^{\prime \prime} F^{\prime \prime \prime}\right|^{1 / 3}}$.

Case IIIn. The similarity reductions take either the form

$u=-\frac{1}{3} p q r t^{3}+\frac{p r}{2} z t^{2}+\frac{1}{2}\left(p \alpha_{1}-\beta q\right) t^{2}+\beta t z+\alpha_{2} t+F(z-q t)+p t G(z-q t)$

$v=-\frac{1}{2} q r t^{2}+r t z+\alpha_{1} t+G(z-q t)$

or

$$
u=K z+q z^{2}+\frac{1}{3} p r z^{3}+F(t)+r z G(t) \quad v=L z+p z^{2}+G(t)
$$

where $p, q, r, \alpha_{1}, \alpha_{2}, \beta, K$ and $L$ are all arbitrary constants. Substitution of (112) into (89)-(90) yields the following equations for the functions $G$ and $F$,

$$
\begin{aligned}
& -q F^{\prime}+p G+\alpha_{2}+\beta \eta=\frac{F^{\prime \prime}}{\left|G^{\prime \prime \prime} F^{\prime \prime}-G^{\prime \prime} F^{\prime \prime \prime}\right|^{1 / 3}} \\
& -q G^{\prime}+\alpha_{1}+r \eta=\frac{G^{\prime \prime}}{\left|G^{\prime \prime \prime} F^{\prime \prime}-G^{\prime \prime} F^{\prime \prime \prime}\right|^{1 / 3}}
\end{aligned}
$$

whereas substitution of (113) into (89)-(90) gives

$$
F^{\prime}=\frac{2 q}{\left|4 r p^{2}\right|^{1 / 3}} \quad G^{\prime}=\frac{2 p}{\left|4 r p^{2}\right|^{1 / 3}}
$$

\section{Conclusions}

We have systematically derived the Lie point symmetry groups of the PDEs associated with the geometric law of motion

$$
\boldsymbol{v}=\phi(\kappa, \tau) \kappa \boldsymbol{n}+\beta(\kappa, \tau) \kappa \boldsymbol{b}
$$


describing the evolution of a space curve. We have shown all laws of this form to be invariant under an arbitrary rotations and translations, that is the action of the special Euclidean group $\mathrm{SE}(3)$. As a corollary, they exhibit rotating travelling wave similarity reductions of the form

$$
r=R(\theta-c t) \quad z=q t+f(\theta-c t)
$$

and helical similarity reductions of the form

$$
r=R(t) \quad z=k \theta+f(t) .
$$

When $\phi=0$, so the velocity law is length preserving, we might expect to find closed curve rotating travelling wave solutions. This phenomenon has been described previously by Kida [7] for the velocity law $\boldsymbol{v}=\kappa \boldsymbol{b}$ which describes the motion of a thin inviscid fluid vortex filament (the full set of similarity reductions to $\boldsymbol{v}=\kappa \boldsymbol{b}$ are identical to those for $\boldsymbol{v}=\kappa \boldsymbol{n}$, which are discussed in [13]). More general isotropic laws of motion (which may also involve an acceleration term) such as the two models for the evolution of scroll waves [9] and elastic rods [10], noted in the introduction, will be invariant under the same Lie point symmetry groups as (114) and thus also display rotating travelling wave and helical similarity reductions.

We have also demonstrated that the subclass of laws of form

$$
\boldsymbol{v}=\kappa^{c+1}\left(\Upsilon\left(\frac{\kappa}{\tau}\right) \boldsymbol{n}+\Gamma\left(\frac{\kappa}{\tau}\right) \boldsymbol{b}\right)
$$

are, in addition, invariant under a rescaling (note that $\boldsymbol{v}=\kappa \boldsymbol{n}$ and $\boldsymbol{v}=\kappa \boldsymbol{b}$ both fall within this subclass). We have used this fact to show that such laws have two extra similarity reductions. When $c \neq-2$ these are

$$
r=t^{1 /(2+c)} R(\theta-p \log t) \quad z=t^{1 /(2+c)} f(\theta-p \log t)
$$

and

$r=(-t)^{1 /(2+c)} R(\theta-p \log (-t)) \quad z=(-t)^{1 /(2+c)} f(\theta-p \log (-t))$

and for $c=-2$ they are

$$
r=R(\theta-c t) \quad z=\exp (\beta t) f(\theta-c t)
$$

and

$$
r=\exp (M \theta) R(t) \quad z=\exp (M \theta) f(t) .
$$

Similarity solutions of the form (116) (or (115)) with $p=0, R=R(t)$ and $f=0$ exist for all such laws, provided that $\Upsilon$ and $\Gamma$ both have finite limits as their arguments tend to $\infty$. Such solutions describe the evolution of a circle. Similarity solutions of the form (116) which describe the shrinking of an 'infinite closed curve' to a point in finite time were constructed in [13] for $\boldsymbol{v}=\kappa \boldsymbol{n}$ and it is likely that these results can be extended to other laws of motion.

A particularly noteworthy result is the identification of the law

$$
\boldsymbol{v}=\left(\frac{\kappa}{|\tau|}\right)^{1 / 3} \boldsymbol{n}
$$

which exhibits a remarkably rich symmetry. It is noteworthy that there is essentially only one member of the family (1) having this special status. They are invariant under a thirteendimensional group corresponding to the general linear group GL(3) acting on $u, v$ and $z$ with a rescaling and translation of $t$. We are unaware of any other PDEs with this invariance. The general linear invariance of these laws means that their solutions preserve information about the shape of a curve ( $\mathrm{cf} v=\kappa^{1 / 3} n$ in two dimensions). The image processing of space curves (see [15] for a discussion of this topic) provides a possible application of this result. In order 
to emphasize the richness of the group structure of (117), we give the complete catalogue of the similarity reductions below:

$$
\begin{aligned}
& u=J z+K v+( \pm t)^{\alpha} F\left(z( \pm t)^{-\beta}\right) \\
& u=J v+K z+\exp (\beta t) F(z \exp (-\alpha t)) \\
& v=L z+( \pm t)^{3-\alpha-\beta} G\left(z( \pm t)^{-\beta}\right) \\
& v=L z+\exp (-(\alpha+\beta) t) G(z \exp (-\alpha t))\} \\
& u=J v+K z+( \pm z)^{\alpha} F(t) \\
& \left.v=L z+( \pm z)^{-(1+\alpha)} G(t) .\right\} \\
& u=J v+K z+( \pm t)^{\alpha} F(z-M \log ( \pm t)) \\
& u=J v+K z+\mathrm{e}^{\alpha t} F(z-c t) \\
& v=L z+( \pm t)^{3-\alpha} G(z-M \log ( \pm t)) \\
& \left.v=L z+\mathrm{e}^{-\alpha t} G(z-c t) \quad\right\} \\
& u=J v+K z+\mathrm{e}^{\alpha z} F(t) \\
& u=J v+K z+( \pm t)^{3 / 2-\beta / 2} \rho\left(z( \pm t)^{-\beta}\right) \cos \left(M \log ( \pm t)-\phi\left(z( \pm t)^{-\beta}\right)\right) \\
& v=L z+q( \pm t)^{3 / 2-\beta / 2} \rho\left(z( \pm t)^{-\beta}\right) \sin \left(M \log ( \pm t)-\phi\left(z( \pm t)^{-\beta}\right)\right) \\
& u=J v+K z+\mathrm{e}^{-\beta t} \rho\left(z \mathrm{e}^{-2 \beta t}\right) \cos \left(M t-\phi\left(z \mathrm{e}^{-2 \beta t}\right)\right) \\
& \left.v=L z+q \mathrm{e}^{-\beta t} \rho\left(z \mathrm{e}^{-2 \beta t}\right) \sin \left(M t-\phi\left(z \mathrm{e}^{-2 \beta t}\right)\right) \quad\right\} \\
& u=J v+K z+( \pm z)^{-1 / 2} \rho(t) \cos (M \log ( \pm z)-\phi(t)) \\
& v=L z+q( \pm z)^{-1 / 2} \rho(t) \sin (M \log ( \pm z)-\phi(t)) \\
& u=J v+K z+( \pm t)^{3 / 2} \rho(z-M \log ( \pm t)) \cos (\Omega \log ( \pm t)-\phi(z-M \log ( \pm t))) \\
& v=L z+q( \pm t)^{3 / 2} \rho(z-M \log ( \pm t)) \sin (\Omega \log ( \pm t)-\phi(z-M \log ( \pm t))) \\
& u=J v+K z+\rho(z-c t) \cos (\Omega t-\phi(z-c t)) \\
& v=L z+q \rho(z-c t) \sin (\Omega t-\phi(z-c t)) \\
& u=J v+K z+\rho(t) \cos (\Omega z-\phi(t)) \\
& v=L z+q \rho(t) \sin (\Omega z-\phi(t)) \\
& u=K z+( \pm t)^{(3 / 2-\beta / 2)}\left[F\left(z( \pm t)^{-\beta}\right)+p \log ( \pm t) G\left(z( \pm t)^{-\beta}\right)\right] \\
& v=L z+( \pm t)^{(3 / 2-\beta / 2)} G\left(z( \pm t)^{-\beta}\right) \\
& u=K z+\mathrm{e}^{\alpha t} F\left(z \mathrm{e}^{2 \alpha t}\right)+p t \mathrm{e}^{\alpha t} G\left(z \mathrm{e}^{2 \alpha t}\right) \\
& v=L z+\mathrm{e}^{\alpha t} G\left(z \mathrm{e}^{2 \alpha t}\right) \\
& u=K z+( \pm z)^{-1 / 2} F(t)+p( \pm z)^{-1 / 2} \log ( \pm z) G(t) \\
& v=L z+( \pm z)^{-1 / 2} G(t) \\
& u=K z+( \pm t)^{3 / 2}[F(z-M \log ( \pm t))+p \log ( \pm t) G(z-M \log ( \pm t))] \\
& v=L z+( \pm t)^{3 / 2} G(z-M \log ( \pm t)) \\
& u=J v+K z-\frac{p q}{2}(\log ( \pm t))^{2}+p(v-r z) \log ( \pm t)+F\left(z( \pm t)^{-3}\right) \\
& v=L z+q \log ( \pm t)+G\left(z( \pm t)^{-3}\right) \\
& u=K z+\frac{p q}{2} z(\log ( \pm t))^{2}+( \pm t) F\left(\frac{z}{( \pm t)}\right)+p( \pm t) \log ( \pm t) G\left(\frac{z}{( \pm t)}\right) \\
& v=L z+q z \log ( \pm t)+( \pm t) G\left(\frac{z}{( \pm t)}\right)
\end{aligned}
$$


$\left.\begin{array}{l}u=-\frac{1}{3} p q r t^{3}+\frac{p r}{2} z t^{2}+\frac{1}{2}\left(p \alpha_{1}-\beta q\right) t^{2}+p t G(z-q t)+\beta t z+\alpha_{2} t+F(z-q t) \\ v=-\frac{1}{2} r q t^{2}+r t z+\alpha_{1} t+G(z-q t)\end{array}\right\}$

$u=K z+\frac{1}{3} r p z^{3}+q z^{2}+r z G(t)+F(t)$

$v=L z+p z^{2}+G(t)$.

A simple but instructive example of a similarity solution to (89)-(90), arising from the velocity law $\boldsymbol{v}=(\kappa /|\tau|)^{1 / 3} \boldsymbol{n}$, takes the form

$$
u=J v+K z+\rho(t) \cos (\Omega z-\phi(t)) \quad v=L z+q \rho(t) \sin (\Omega z-\phi(t))
$$

which gives rise to the following equations for $\rho$ and $\phi$ :

$$
\rho^{\prime}=-\frac{\rho^{1 / 3} \Omega^{2}}{\left|q \Omega^{5}\right|^{1 / 3}} \quad \rho \phi^{\prime}=0 .
$$

These have solution

$$
\rho=\left(\frac{2 \Omega^{2}}{3\left|\Omega^{5} q\right|}\right)^{3 / 2}\left(t_{0}-t\right)^{3 / 2} \quad \phi=\phi_{0}
$$

where $t_{0}$ and $\phi_{0}$ are constants of integration. An animation of the similarity solution of this form can be found on the web [14]. The noteworthy feature of this solution, which describes the shrinking of a uniformly strained helix, is that the curve retains features of its shape as it shrinks towards a straight line. So if, for example, the helix is projected onto a plane perpendicular to its axis an ellipse results; this ellipse retains its eccentricity as the spiral shrinks.

Finally as in [13], we conclude by giving a quite different application of our results (to coupled nonlinear diffusion equations) which pertains to the earlier comment that the above symmetries are far from obvious from the PDE formulation. Thus, writing

$$
\alpha=\frac{\partial u}{\partial z} \quad \beta=\frac{\partial v}{\partial z}
$$

the law of motion

$$
\boldsymbol{v}=\kappa(\boldsymbol{n}+\mu \boldsymbol{b})
$$

where $\mu$ is a constant leads (via (6)-(7)) to the nonlinear cross-diffusion system

$$
\begin{aligned}
& \frac{\partial \alpha}{\partial t}=\frac{\partial}{\partial z}\left(\left(\frac{1}{1+\alpha^{2}+\beta^{2}}+\mu \frac{\alpha \beta}{\left(1+\alpha^{2}+\beta^{2}\right)^{3 / 2}}\right) \frac{\partial \alpha}{\partial z}-\frac{\mu\left(1+\alpha^{2}\right)}{\left(1+\alpha^{2}+\beta^{2}\right)^{3 / 2}} \frac{\partial \beta}{\partial z}\right) \\
& \frac{\partial \beta}{\partial t}=\frac{\partial}{\partial z}\left(\left(\frac{1}{1+\alpha^{2}+\beta^{2}}-\mu \frac{\alpha \beta}{\left(1+\alpha^{2}+\beta^{2}\right)^{3 / 2}}\right) \frac{\partial \beta}{\partial z}+\frac{\mu\left(1+\beta^{2}\right)}{\left(1+\alpha^{2}+\beta^{2}\right)^{3 / 2}} \frac{\partial \alpha}{\partial z}\right) .
\end{aligned}
$$

This has a rich family of (partially non-local) symmetries corresponding to the point symmetries of (118) and is a special case of the class of cross-diffusion systems

$$
\frac{\partial \phi}{\partial t}=\nabla \cdot\left(D_{1}(|\phi|)+\mathrm{i} D_{2}(|\phi|) \nabla \phi+\left(D_{3}(|\phi|)+\mathrm{i} D_{4}(|\phi|)\right) \phi \nabla|\phi|\right)
$$

where $\phi=\alpha+\mathrm{i} \beta$ and the $D_{i}$ are real, which has a local rotation symmetry in $\phi$ (i.e. is invariant under translations in $\arg (\phi))$. Moreover, by setting

$$
\beta=\mathrm{i} b \quad \mu=\mathrm{i} v
$$


in (119) we obtain

$$
\begin{aligned}
& \frac{\partial \alpha}{\partial t}=\frac{\partial}{\partial z}\left(\left(\frac{1}{1+\alpha^{2}-b^{2}}-v \frac{\alpha b}{\left(1+\alpha^{2}-b^{2}\right)^{3 / 2}}\right) \frac{\partial \alpha}{\partial z}+\frac{v\left(1+\alpha^{2}\right)}{\left(1+\alpha^{2}-b^{2}\right)^{3 / 2}} \frac{\partial b}{\partial z}\right) \\
& \frac{\partial b}{\partial t}=\frac{\partial}{\partial z}\left(\left(\frac{1}{1+\alpha^{2}-b^{2}}+v \frac{\alpha b}{\left(1+\alpha^{2}-b^{2}\right)^{3 / 2}}\right) \frac{\partial b}{\partial z}+\frac{v\left(1-b^{2}\right)}{\left(1+\alpha^{2}-b^{2}\right)^{3 / 2}} \frac{\partial \alpha}{\partial z}\right)
\end{aligned}
$$

while setting

$$
\alpha=\mathrm{i} a \quad \beta=\mathrm{i} b
$$

gives

$$
\begin{aligned}
& \frac{\partial a}{\partial t}=\frac{\partial}{\partial z}\left(\left(\frac{1}{1-a^{2}-b^{2}}-\mu \frac{a b}{\left(1-a^{2}-b^{2}\right)^{3 / 2}}\right) \frac{\partial a}{\partial z}-\frac{\mu\left(1-a^{2}\right)}{\left(1-a^{2}-b^{2}\right)^{3 / 2}} \frac{\partial b}{\partial z}\right) \\
& \frac{\partial b}{\partial t}=\frac{\partial}{\partial z}\left(\left(\frac{1}{1-a^{2}-b^{2}}+\mu \frac{a b}{\left(1-a^{2}-b^{2}\right)^{3 / 2}}\right) \frac{\partial b}{\partial z}+\frac{\mu\left(1-b^{2}\right)}{\left(1-a^{2}-b^{2}\right)^{3 / 2}} \frac{\partial a}{\partial z}\right) .
\end{aligned}
$$

All of these systems will possess extensive symmetry groups and a correspondingly wide range of similarity reductions; none of them would be likely to be identified as special cases of cross-diffusion systems purely by inspection and (120), (121) lack a direct geometrical interpretation (in contrast to the equivalence of (119) to (118)). Further generalizations along the lines of those described in [13] for $\mu=0$ are also possible.

\section{Acknowledgment}

GR gratefully acknowledges the support of a Leverhulme special research fellowship.

\section{References}

[1] Special Issue 1997 Vortices, dislocations and line singularities in partial differential equations Phil. Trans. R. Soc. A 355 1731, 1945-2072

[2] Bluman G W and Cole J D 1974 Similarity Methods for Differential Equations (New York: Springer)

[3] Bluman G W and Kumei S 1989 Symmetries and Differential Equations (Berlin: Springer)

[4] Callegari A J and Ting L 1978 Motion of a curved vortex filament with decaying vortical core and axial velocity SIAM J. Appl. Math. 35 148-75

[5] Chapman S J and Richardson G 1995 Motion of vortices in type-II superconductors SIAM J. Appl. Math. 55 1275-96

[6] Hydon P E 2000 Symmetry Methods for Differential Equations (Cambridge: Cambridge University Press)

[7] Kida S 1981 A vortex filament moving without change of form J. Fluid Mech. 112 397-409

[8] Lin T 2000 Rigorous and generalized derivation of vortex line dynamics in superfluids and superconductors SIAM J. Appl. Math. 60 1099-110

[9] Keener J P and Tyson J J 1992 The dynamics of scroll waves in excitable media SIAM J. Appl. Math. 34 1-39

[10] Klapper I and Tabor M 1994 A new twist in the kinematics and elastic dynamics of thin filaments J. Phys. A: Math. Gen. 27 4914-24

[11] Moore D W and Saffman P G 1972 The motion of a vortex filament with axial flow Phil. Trans. R. Soc. A 272 403-29

[12] Olver P J 1995 Application of Lie Groups to Differential Equations (Berlin: Springer)

[13] Richardson G and King J R 2002 Motion by curvature of a three-dimensional filament: similarity solutions Interfaces Free Bound. 4 395-421

[14] Richardson G 2002 webpages http://www.maths.nottingham.ac.uk/personal/etzgwr/affinemotion.html and stacks.iop.org/JPhysA/35/9857

[15] Salden A H, ter Haar Romeny B M and Viergever M A 1999 Linearised Euclidean shortening flow of curve geometry Int. J. Comput. Vis. 34 29-67

[16] Sapiro G and Tannenbaum A 1992 Affine invariant scale space Int. J. Comput. Vis. 11 25-44

[17] Wood G P 1996 Some problems in nonlinear diffusion PhD Thesis University of Nottingham 